\title{
Dividing by Zero. Tautology and Paradox in the Self-Descriptions of Anonymous
}

\author{
Davide Beraldo \\ University of Amsterdam
}

This paper engages with the loosely bounded, ill-defined Anonymous movement, in order to develop a theoretical reflection on the process of self-reference within contemporary collectives. It is grounded on a socio-cybernetic framework and builds on a computationally-assisted interpretative analysis of a huge dataset of Facebook posts related to Anonymous' self-descriptions. As selected examples show, Anonymous results inherently contradictory. Its boundaries are radically contingent and performative, to the extent that it is impossible to distinguish the authentic from the inauthentic. The collective defines itself as ontologically multiple and radically anti-essentialist. Moreover, whereas Anonymous' actions are systematically contradictory, Anonymous self-descriptions, relying on arguments mirroring poststructuralist theories, can only be tautologically or paradoxically expressed. Building on Luhmann's claim that the reproduction of modern societies depends on concealing their self-referential foundations, the conclusion argues that Anonymous, by embracing its own constitutive paradox, pushes the process of autopoiesis to a new, radically recursive logic, departing in this even from recent theorizations on reflexivity.

url: https://papiro.unizar.es/ojs/index.php/rc51-jos/article/view/4246

\author{
،“'Everything said is said by an observer' an \\ observer said' an observer said' an observer said'
}

(variation on Maturana and Varela, Autopoiesis and Cognition: The Realization of the Living, 1980, p. 8)

\section{Introduction}

Anonymous, a loosely associated network of online contention (Coleman, 2015; Olson, 2013), presents a multifaceted personality. Despite being closely tied to an emergent 'geek culture' associated with hackers, tech-savvies and creatives (Coleman, 2013), radically different political (and non-political) worldviews coexist behind its mask (C. Fuchs, 2013). Over the years, while alternating phases of concentration and diffusion (Uitermark, 2017), Anonymous has nonetheless maintained a substantial, however fluid, organizational identity (Dobusch \& Schoeneborn, 2015). How can this 
highly contradictory, unstable and conflictual system reproduce itself as a distinct and recognizable entity, instead of splitting, jeopardizing or imploding under the push of its several 'schismogenetic' tendencies? This paper tries to solve this puzzle by investigating Anonymous' self-descriptions building on a second-order cybernetic framework (Bateson, 1972; Luhmann, 1995; Maturana \& Varela, 1991). The central claim is that Anonymous' relation with complexity is key to understanding its reproduction beyond contradictions: by embracing its own structural paradox at the level of the semantics, instead of resolving it as social systems are generally required to (Luhmann, 1988; Schoeneborn, 2011), Anonymous manages to be 'radically recursive' (Rosen, 2004); in this, the collective seems to depart even from the radically reflexive models typically associated with late modernity (Beck et al., 1994; Dean, 2009; Melucci, 1996).

Evolutionary unpredictability, ideological diversity, and structural complexity are common features of contemporary digitally-networked, 'multitudinous' movements (Bennett \& Segerberg, 2012; Chesters \& Welsh, 2005; Monterde et al., 2015); nonetheless, Anonymous stands out because, in spite of manifesting all these characteristics to an extreme extent, it managed to reproduce a recognizable organizational identity for more than a dozen years. Hence, it provides a quasi-idealtypical case through which to reflect on identity formation and systemic reproduction under the paradoxical conditions fostered by digital communication.

The paper is structured as follow. A theoretical section briefly introduces key notions of second-order cybernetics and highlight the puzzle posed by Anonymous. A methodological section explains the rationale of adopting Facebook's data to point out key-aspects of Anonymous' semantics and self-descriptions. By means of few illustrative examples, the following section demonstrates how Anonymous is marked with systematic contradiction. Further material illustrates the continuously performative and radically contingent character of Anonymous' boundaries production, from which descends the impossibility of discerning between the Anonymous-original and the Anonymous-copy. Subsequently, paradigmatic declarations on Anonymous' nature and definition demonstrates how its anti-foundational and anti-essential traits are sometimes overtly represented, rather than concealed, in its semantics. Departing from the observation that complexity is already unveiled by Anonymous' own self-descriptions, the conclusion moves forward arguing that Anonymous represents a fringe social formation, one that recursively reproduces itself by abstracting a meta-layer of self-descriptions on a higher degree of abstraction than that of classical (modern) social systems. This model seems to deviate even from recent theorization on reflexivity (Beck, Giddens, \& Lash, 1994; Chesters \& Welsh, 2005; Dean, 2009; Melucci, 1996). In order to mark this departure, a distinction between 'radical reflexivity' (i.e. a form of self-referentiality that integrates a system through the continuous synthesis of new definitions) and 'radical recursivity' (i.e. a form of self-referentiality that integrates a system through the continuous abstraction of new meta-levels) is proposed, arguing that the latter is better-suited at capturing the peculiar self-referential logic foregrounded by Anonymous' autopoiesis.

\section{Self-reference in Anonymous}

It is extremely challenging to grasp Anonymous' ontological status, which is somewhere in between categories such as movement, network, swarm and multitude (Wiedemann, 2014). In this paper, the question of 'what is Anonymous' is set aside, and the focus is on 'who is Anonymous' instead, as an entry-way to assess its surprising capacity to reproduce its organizational identity (i.e. its persistence as a recognizable functional system) besides its fundamental contradictions. Coherently with a cybernetic / systemic approach, Anonymous is conceptualized here as a system that communicatively self-reproduces its distinction from the environment (who is not Anonymous), 
realizing its autopoiesis through the deployment of a number of constitutive self-descriptions (Luhmann, 1988, 1995). Considering its radical fluidity and its nature of (mostly) online-only phenomenon, Anonymous represents a radical case from where to understand organization as an outcome of communication systems (Dobusch \& Schoeneborn, 2015; Kavada, 2015; Schoeneborn, 2011).

Autopoiesis is the capacity of systems to self-produce their organizational identity (Maturana \& Varela, 1991); this capacity depends on the self-referential (recursive) character of these systems: the rules of interaction among the elements of a system incorporate the conditions for the reproduction of the system as a whole. Conversely, schismogenesis is the self-sustained process that leads to the progressive differentiation of a system into more and more autonomous subsystems and that, if not counterbalanced, ultimately leads the system to fall apart (Bateson, 1972). The autopoiesis of social system, according to Luhmann, is realized by self-observations (i.e. self-referential distinctions the system makes between the system itself and its outside) operating in the domain of communication (Luhmann, 1992). Self-descriptions, more in particular, are the set of self-referential observations of social systems upon which their reproduction critically depends. These self-descriptions, responsible for defining the identity of a social system, can only be expressed on a fundamental level as tautologies or as paradoxes (Luhmann, 1988). However, 'modern society does not admit that its self-description faces a problem of tautology or paradox' (Luhmann, 1988, p. 28); thus, recursive self-descriptions need to be 'detautologized' and/or 'deparadoxified' so as to 'offer specific descriptions of society and recommend particular programs for actions' (Luhmann, 1988, p. 32). Ideologies, specifically, represent the development of self-descriptions in non-recursive self-observations, hiding their original tautological or paradoxical (thus, self-referential / recursive) character.

The contradictory traits of Anonymous should be expected to act as a catalyst for schismogenesis. While recognizing themselves as part of the same systemic entity, different Anonymous branches are aware of embracing contrasting causes and ideologies; this can generate incompatible self-observations and, as clear from the sections below, the impossibility to resolve the self-referential character of Anonymous' definitions. We would then expect each of the different branches to develop its own self-definition and, in perspective, its own distinct identity. To the contrary, Anonymous manifests a persistent counter-schismogenetic property by preserving, instead of developing, the self-referential character of its descriptions: contradiction is maintained and (on another level) resolved into paradox, thus affording autopoiesis rather than contributing to schismogenesis. By refusing to detautologize or deparadoxize its self-descriptions, Anonymous embraces what we can call, after Rosen (2004), 'radical recursion', a form of recursivity that 'goes all the way down', in that the self-referential nature of the system is explicitly incorporated in its semantics instead of concealed, thus generating a further meta-level of abstraction. Whereas in reflexive collectives identity is continuously reconstructed as a synthesis of heterogeneous, interactive positions (Chesters \& Welsh, 2005; Melucci, 1996), and in a context where the exacerbation of reflexivity is said to generate endless loops of questioning and doubts (Dean, 2009; Mumby, 2016), Anonymous should be interpreted instead as radically recursive, in that it abstracts a meta-level of self-definitions doing away with the need for any reflexive synthesis. By anticipating its own 'schizophrenic' character, that is to say by refusing to stabilize its identity so as to escape any apparatus of capture (Deleuze \& Guattari, 1987), the conditions of Anonymous' dismantling are turned into the paradoxical conditions of its tautological reproduction.

\section{Investigating Anonymous' Semantics on Facebook}


Following perspectives that understand organization as an inherently communicative phenomenon (Luhmann, 1992; Schoeneborn, 2011), the above mentioned questions are answered looking at textual data produced by Anons. Inspired by interpretative content analysis (Ahuvia, 2001) and semantics analysis a(Andersen, 2011), the research turned to Facebook as a repository of data, using Facebook's API as an extraction tool to collect textual data from public pages (Rieder et al., 2015).

In operative terms, this paper investigates Anonymous by looking at digital traces related to textual self-descriptions, focusing on three levels of definitions: examples of goals, political orientations and worldviews displayed in Anonymous' discourse (ostensive definition); the production of the boundaries between authentic/inauthentic utilizations of the Anonymous' identity (extensional definition); and claims on the essence and ontological status of the collective (intensional definition). Consequently, the following sub-questions are posed: a) Who is Anonymous? In the sense of: Which orientations, ideologies, goals lie behind the adoption of the label 'Anonymous'? b) Who is Anonymous? In the sense of: Which criteria make an individual or collective actor an (in)authentic member of Anonymous? c) Who is Anonymous? In the sense of: What are the defining elements of Anonymous' essence?

The choice to focus on such a 'nonymous' medium like Facebook might sound at odds with the very nature of Anonymous. However, there are both substantial and convenience reasons to justify this operative choice. Firstly, and most importantly, Facebook pages were the most reasonable choice as the goal of this research was to focus on self-descriptions, in that they provide a historical repository of textual data, often in the form of pages administrators representing the group towards the readers and of the subsequent reactions of user participating in the page; other relevant platforms more typically associated with Anonymous, such as IRC channels or Twitter, would be better suited to study other aspects such as decision-making or structural relations among different branches. Secondly, whereas Anonymous originated on more obscure corners on the Web such as 4chan and IRC channels, its social media presence became stunning together with its popularization brought by mainstream media coverage; this is confirmed by the results of this study, as the iterative crawling procedure employed retrieved almost 6 thousands pages, many of which with a substantial following (the top 10 pages exceeds 100,ooo likes). Thirdly, at the time of this research, Facebook APIs allowed for the large-scale and far-reaching data collection required by the overall research project, thus providing a strong point in terms of research pragmatism.

The data collection consisted of three main steps: first, a set of Facebook pages related to Anonymous was collected; second, the network of 'like' relationships between these and toward other pages was built; and third, the posts, comments and replies contained in these pages were stored in a database for textual analysis. The selection of pages thus exploited an iterative crawling procedure. Facebook's Graph API was pulled for pages containing the keyword 'anonymous', which resulted in the retrieval of 500 pages. Starting from this seeding sample, the API was queried to get 25 pages that were 'liked' by each of the pages previously obtained, retaining all the pages containing the sub-string 'anon' within their name. In this way, it was possible to expand the initial sample. The procedure just described was reiterated until the query did not return any new pages containing the substring 'anon' within its name. Subsequently, the list of pages was manually checked to filter out anything obviously off-topic. In so doing, a list of 5,777 Facebook pages relating to Anonymous was obtained.

Facebook pages are commonly articulated around content posted by page administrators or users liking the page, often generating chains of comments. This provides access to a vast amount of textual data. Facebook's Graph API was subsequently interrogated to retrieve posts, comments and 
replies associated with the list of Anonymous pages collected. Queries were run between February and March 2016, and thus reflect the state of Anonymous' presence on Facebook at that time. A total of 1,328,714 strings of text were consequently collected, spanning between November 2008 and April 2016. This massive corpus has been queried with an iterative interpretative procedure to identify traces of collective self-descriptions, following a logic of purposive sampling (Palys, 2008). Posts and comments deemed particularly useful for illustrating the argument are reported in the respective section.

The selection of the empirical material presents obvious limitations in terms of representativity of Anonymous as a whole; however, the goal of this paper is conceptual. Whereas Anonymous' organization is realized in several settings other than Facebook, the excerpts reported here are selected examples of recurrent self-descriptions that point out the relevance of (explicit) paradoxes and tautologies.

The following sections interpret the textual data retrieved from Anonymous' Facebook pages as traces of Anonymous self-descriptions, showing how, by incorporating complex ontological statements within its own semantics, Anonymous exploits its radically recursive character to transform contradiction into paradox, thus turning schismogenetic factors into a source of autopoiesis.

\section{Who Is Anonymous? Contradictory Orientations}

Before initiating a systematic collection of Facebook pages through API calls, the investigation started with a phenomenological exploration of Anonymous, exploiting Facebook's recommender algorithm. A Facebook user was created for the purpose, without any previous 'like' experience, and the strategy was simply to input the term 'anonymous' in the search bar and scroll through the suggested results, liking every single page bearing reference to Anonymous. The result was a Facebook feed characterized by a cacophonic stream, in which reports on leaks against authoritarian governments alternated with alleged evidence on the involvement of Prince Andrew, Duke of York, in a ritualistic pedophile ring; Islamist propaganda against Jewish people and the so-called 'Holocaust-industry' was followed by reports on the supposedly miraculous effects of Marijuana on brain tumors; and information on privacy loopholes in the latest Android update coexisted with claims of proved contact between major world governments and alien entities.

Anonymous nowadays makes an appearance in almost every critical political scenario. Wherever and whenever people take to the streets to protest against corruption, abuses or misconduct of all sorts perpetrated by a government of any color, there is a good chance that one or multiple Anonymous operations will pop up. Taking 2013 Ukraine crisis as a test case, it is evident that the Anonymous label was taken up both in support of the 'legitimate revolt of the Ukrainian people against the corrupted puppets led by Moscow' and to 'fight back against the NATO-backed para-fascist coup'. It is worth to stress that this is only one among the several analogous examples of plainly contradictory positions taken up by Anonymous in relation to major geopolitical issues. The following opposing declarations, both claiming to represent Anonymous' voice on the issue, are paradigmatic of this trait:

Greetings Citizens of the World, We are Anonymous Ukraine. [...] The people of Ukraine do not want European Union integration. The people of Ukraine do not want NATO on their territory. The people of Ukraine voted for President Yanukovich to lead them in fair and just 
democratic elections. The people of Ukraine plea to the President and to Russia for help in stopping the siege of Ukraine by Nazi thugs and murderous gangs.

(page name: Anonymousarmy, page ID: 444851598955819, post ID: 701338436555383, date: 10 March 2014)

To People of the Ukraine, we are anonymous. To politicians, police, and the government of Ukraine as an entity, Anonymous, is watching the revolution in your country unfold. We are paying close attention to the brutal \& barbaric police suppression with which these demonstrations are met, with ever growing concern and rising anger. [...] When President Viktor Yanukovych caved in to pressure from Russia and abandoned the EU agreement a week before it was to be signed, he showed contempt for Ukrainian voters and a craven inability to stand up for himself, his government, or the democracy he claims to represent.

(page name: Anonymous - EU Bulgaria, page ID: 422706107791268, post ID: 612201268841750, date: 6 December 2013)

Anonymous generally professes itself as beyond conventional ideologies, and as an alternative to any political party or candidate. As Anons themselves put it:

THE IDEOLOGY OF ANONYMOUS: Anonymous does not have a political agenda and no political ideology party. ANONYMOUS HAS MORAL IDEOLOGIES: The moral ideologies are freedom

(page name: Anonymous \#OpStopCorruption; page ID: 172486642949943; post ID: 284396548425618L, date: 5 November 2014)

The concrete adoption of the Anonymous identity, however, often contravenes this rule. Anonymous, implicitly or explicitly, does take sides on political issues. Scanning the list of pages, indeed, we find a vast array of references to specific political ideologies such as anarchism, libertarianism and communism. Whereas some European branches of Anonymous openly adhere to a clearly left-wing declination of anarchism (engaging with traditional leftist issues such as anti-fascism, migration rights and anti-capitalism), in the United States Anonymous has also become intertwined with the well-established right-wing libertarian culture associated with the patriotic activism of the so-called 'militia movement'. These two movements share concerns about intrusive governments and big corporations, though their world vision could not be more divergent. An interesting case is that of 'Anonymous Patriots', juxtaposing the symbolism of Anonymous with themes associated with a strong American nationalistic rhetoric, for example the right of individuals to arm themselves to defend their properties, the fear of a socialist degeneration of US big government, and the call to stop a migrant invasion.

Whereas the notion of identity is often associated with homogeneity and coherence, constructivist perspectives on collective identity specify that identity is to be understood as the dynamic outcome of negotiation and even conflict (Melucci, 1996). Many recent social movements present an highly heterogeneous character, which led some scholars to question their status of 'collectives' (Bennett \& Segerberg, 2012; McDonald, 2006). Nonetheless, if we understand the construction of a collective as an inherently communicative process (Kavada, 2015; Schoeneborn, 2011), we cannot but conclude that Anonymous does possess an element of organizational identity (Dobusch \& Schoeneborn, 2015). Its boundaries, indeed, are continuously reproduced: implicitly, in terms of the standardized adoption of strong identity markers (the name Anonymous, its symbolism 
and its slogans); and explicitly, in so far as certain definitions of who is Anonymous and who is not are frequently produced. Whereas this section has illustrated how an ostensive definition of Anonymous, one based on illustrating a number of illustrative examples, is deemed to fail, the following section focuses on the extensive definitions associated with what, according to participants, makes certain actors or actions 'authentic' expressions of Anonymous.

\section{Who Is Anonymous? Controversies of (In)authenticity}

This section focuses on the recurrent controversies present in the corpus, connected with the question of who - individual or collective actor - is 'really Anonymous' and can thus legitimately claim its identity. This means observing cases of discursive production of the boundaries of Anonymous.

Chronologically speaking, the first and most evident line of division is that between the original 'lulz' character of earlier Anonymous activity and the subsequent emergence and prevalence of political activism. The distinction between the political and the irreverent uses of the Anonymous label often accompanies inauthenticity claims.

There is nothing political in what we do, we do it for the lulz anything else is just fail.

(page name: Anonymous; page ID: 389916374410483; post ID: 3928809; 5 July 2013)

This is the real anon. All the newfags that don't comprehend this; GTFO off this anon page, go like one of the newfag pages. [...] the only thing not legit is anon in it's current form.

(page name: Anonymous; page ID: 149062061842666; post ID: 3058652; date: 29 July 2012)

The modalities in which an Anonymous operation emerges are most likely multiple; however there is a distinctive model of decision making in line with the idea of Anonymous as an affinity group with an orientation which can be redirected according to contingent factors. The legitimacy of an appropriation, as the following statement makes explicit, depends on whether Anons agree among themselves that the appropriation is legitimate.

anyone can launch a new ideological message or campaign under the banner of ANONYMOUS. Anyone can take up a leading role in the spreading of the ANON-consciousness. Whether or not these appropriations are legitimate is decided by the rest of the internet citizenry. [...] if the people do not agree with these actions, then these actions are - by definition - not undertaken by ANONYMOUS.

(page name: We need Anonymous; page ID: 24093564934013; post ID: 41353201867745; date: 22 May 2012)

Disagreements, however, are the norm rather than the exception. This often leads Anons to admit that 'Anonymous is not unanimous': Anons can disagree among themselves, and there is no need for Anonymous to have a coherent opinion.

its called Anonymous not unanimous the amount of infighting amongst anons would make you question if there were even a group to begin with 
(page name: Anonymous; page ID: 625777917578158; post ID: 625821524240464; date: 27 February 2016)

This gives Anonymous the peculiar ability to reproduce itself as many things at once, escaping attempts of annihilation perpetrated by its enemies

Anonymous constantly evolves and changes to how people want it to be. In the end, Anonymous can only be constructed or dismantled within our own imagination, not through arrests and/or threats.

(page name: Anonymous College of Hacktivism; page ID: 19163062091025; post ID: 441657; date: 27 August 2013)

Fascinated by its growing popularity, but confused by its shady nature, a number of people manifest their intention to embrace Anonymous' cause and thus ask the crucial question: how can I join Anonymous? As often explained by Anons, however, there is no such a thing as a membership of Anonymous.

You can not join Anonymous. Nobody can join Anonymous. Anonymous is not an organization. Nobody can speak for Anonymous. Nobody could say: you are in, or you are out. Do you still want to join Anonymous? Well, you are in if you want to.

(page name: Anonymous Germany; page ID: 201170586586592; post ID: 129862327089030; date: 9 May 2011)

Nonetheless, websites and Facebook pages often present instructions on how to get into the 'hive'. A widespread, general-purpose operation, \#OpAnonBlood, states its mission to be the creation of protocols and procedures to introduce interested people into the logic and practices of Anonymous.

Despite the existence of 'inauthenticity claims' as a constant, Anons often state that everyone can be Anonymous - or even that no one is Anonymous.

Anonymous does not have members or leaders. there is no way to get into Anonymous. Anyone who believes in the motto is Anonymous. Period.

(page name: Anonymous; page ID: 22011355801277; post ID: 381097; date: 28 December 2011)

anonymous is no one. anonymous is everyone.

(page name: We need Anonymous; page ID: 24093564934013; post ID: 48945605441421; date: 29 June 2012)

The criteria occasionally mentioned for defining who is part of Anonymous are extremely general, allowing for potentially anybody, without any predefined characteristic, to identify as part of the family.

There is no group to join, If ur questioning a corrupt government. .u $\mathrm{r}$ legion. ..if $\mathrm{u}$ defend the defenseless. .u $r$ legion. ..if u still have a heart and soul ..u $r$ legion..if u believe in a 
better world...u $\mathrm{r}$ legion. .if $\mathrm{u}$ talk to others about ur doubts. .u $\mathrm{r}$ legion..no joining a club. .or following a leader..for $\mathrm{u} r$ the club...and the leader...anonymous is an idea..and $\mathrm{u}$ cant kill an idea. EXPECT US.

(page name: Anonymous Germany; page ID: 20117058658659; post ID: 12986232708903; date: 9 May 2011)

The fact that everybody can potentially be Anonymous paradoxically implies that nobody can claim to be 'more Anonymous' than others. In some cases, indeed, claiming to be really Anonymous creates the conditions for not being really Anonymous.

Anyone can be Anonymous, yet not everyone is Anonymous. [...] we do have a warning, you must be aware of fake Anons, who profit from the name of Anonymous, those who call themselves \OFFICIAL \

(page name: We Are Anonymous; page ID: 27392591608998; post ID: 57939729554284; date: 5 February 2016)

All the points raised above make it really complicated to discern what is official and what is fake within Anonymous. Indeed, Anons often reject the idea that something in Anonymous can be official at all.

Nothing is official. No videos. No operations. Not even this press release, even though it was created by an Anonymous number of Anonymous at an Anonymous time in an Anonymous place and uploaded Anonymously, it does not speak for Anonymous.

(page name: Anonymous Croatia; page ID: 39344785401472; post ID: 53610232308260; date: 10 October 2012)

However, many Anonymous pages resort to the appellative 'Official', seemingly because of the practical need to underline their authenticity among a vast population of other pages. Those pages explicitly claiming to be the official page of Anonymous attracts claims of inauthenticity precisely for their claim of authenticity.

Welcome to the official Melbourne Anonymous facebook fan page.

(page name: Melbanon - (Anti-Scientology Protests); page ID: 19972541670459; post ID: 18849042451852; date: 15 February 2011)

Every time you see something saying 'Official Anonymous', or 'Anonymous Official', or 'Headquarters', you are dealing with real fakes, aka greedy ones, infiltrators, and all things described by us.

(page name: Anonymous Brabant Operations; page ID: 162071183150518; post ID: 170849238939379; date: 11 February 2016)

These examples make clear how Anonymous' cannot but be understood as a discursive construction built upon conflicting 'identity claims' (Dobusch \& Schoeneborn, 2015). More in particular, as shown, Anonymous' identity is continuously at the center of controversies made visible by recurrent 'inauthenticity claims' among its (non-)members. The resulting logic of authenticity is often either tautological (everyone is Anonymous) or paradoxical (no one is Anonymous); moreover, for almost every authenticity rule deemed as essential, there are many cases of violation, 
introducing a further level of contradiction. The practical effect of all this is the recurrent impossibility of discerning between real and fake appropriations: the categories of authentic and inauthentic fail to delimit the boundaries of who is really Anonymous. Still, inauthenticity and authenticity claims are widespread in Anonymous' self-definitions: everything can be either official or fake, according to contingent factors. The Anonymous-original and the Anonymous-copy, in other words, are sometimes indistinguishable because, like simulacra (Baudrillard, 1994), each copy has no reference to any real original. Everyone is Anonymous, because no one is Anonymous; however, as the examples clearly show, someone turns out to be not so Anonymous. The boundaries between 'who is in' and 'who is out' are undefined; but still they are continuously defined. Anonymous is suspended in a continuous, explicit process of group formation: the collective is the situated and contingent accomplishments of the unfolding of controversies (Latour, 2005). Any definition of in-group boundaries is to some extent subjected to contestation and generates accuses of false attributions. However, the intensity and persistence of inauthenticity claims within Anonymous are almost unique in their character and can be understood as a constitutive element of Anonymous' peculiar identity.

If we have to answer the question 'who is Anonymous?', the following statements, all expressed as tautologies or paradoxes, clearly descend from Anons' own definitions reported above:

'Anonymous is who wants to be Anonymous'. 'Anonymous is who Anonymous wants'.

'Anonymous is not unanimous, Anonymous has one voice'. 'You cannot join Anonymous, you are Anonymous'. 'No one is Anonymous, everyone is Anonymous (but someone is not Anonymous)'. 'Official announcement: there are no official announcements'

\section{Who Is Anonymous? Essential Inessentiality}

This section intends to dig into the corpus of Anonymous' posts and comments for insights concerning its ontological status: who is Anonymous, in the sense of what are the constitutive elements of Anonymous' identity?

We are one. We are many. One does not speak for many. Many do not speak for all. No one speaks for all. We come from all walks of life. All age, race, sex, places. We have no ideologies. We have every ideology imaginable. We have no goals. We have every goal imaginable. To understand Anonymous would be to understand our universe. Anonymous cannot be labeled, blamed or made a scapegoat.

(page name: Anonymous News And More; page ID: 48742004463265; post ID: 49110648759734; date: 18 January 2013)

Being simultaneously one and many allows Anonymous to be nothing and everything, to endorse any cause and not to have to endorse any cause. Thus, it allows Anonymous to escape any definition or classification. In other words, as one of its prominent slogan states, Anonymous 'is legion'.

Anonymous is Legion, for we are many

(page name: Anonymous; page ID: 27893179991; post ID: 29466973573; date: 31 January 2010)

This expression comes from the Gospels, where Jesus confronts a man possessed by demons: 'And he asked him, What is your name? And he answered, saying, My name is Legion: for we are many'. Anonymous explicitly refuses to meet this request, becoming the omni-inclusive, ungraspable identity that it proves to be. 
The fact that Anonymous is a legion crossed by continuous conflicts, however, does not mean that it does away with a sense of unity. On the contrary, references to its 'we'-ness and emphasis on unity are extremely frequent:

Anonymous is Legion, for we are many

(page name: Anonymous; page ID: 27893179991; post ID: 29466973573; date: 31 January 2010)

One great thing from our previous attacks was successful because WE speak as one. [...] WE are one, like our Mask represents. No colors, no heights, no religion and no regions. WE are all the same. WE speak as one. WE are family, my brothers...sisters... let us respect each other; WE must unite and meet our common goal and deliver our message. The more WE used WE, the stronger WE are, the weaker they become. WE are Anonymous and WE are family.

(page name: Anonymous Pangasinan; page ID: 14124528270273; post ID: 26199480396111; date: 24 February 2014)

The contradiction in terms that follows the incredibly conflictual character of Anonymous and its emphasis on unity is sometimes interpreted in the sense that Anonymous is the one voice of many voices:

we are not an entity, we are the union of all .. do not fight for a cause, fight for the cause of all (page name: Anonymous Grupo; page ID: 21788375834254; post ID: 27858206893937; date: 8 February 2013)

In this way, Anonymous can fit divergent positions and empower all of them: it gets stronger and stronger precisely by incorporating any aspect of the world, by collecting many voices under the same name, and providing them with an element of unity beyond the differences.

Looking for the essential traits defining Anonymous' identities means to elucidate the ontological status of Anonymous. The following post provide Anonymous' perspective on this:

we'd like to take this opportunity to elucidate our goals, ideology and ontological status. First and foremost, it is important to realize that ANONYMOUS - in fact - does not exist.

ANONYMOUS is nothing but an idea

(page name: Anonymous Ohio; page ID: 48464697490774; post ID: 48465072157403; date: 6 January 2013)

Anonymous, in Anons' own words, does not exist. The fact that Anonymous is nothing, however, implies that Anonymous can be everything. When Anons have to concretely define what Anonymous is, they often say it is not a group or organization but an idea, something more abstract than anything that really exists.

Anonymous is nobody. Anonymous is nothing. Anonymous is an idea. Ideas cant be killed (page name: Anonymous Nation; page ID: 44666233209313; post ID: 45043579171578; date: 24 April 2013) 
For this very reason, Anonymous is immortal, because ideas do not belong to the actual world, but resist in a state of virtuality. They cannot be annihilated, because they really are without actually being.

Anonymous has no face, no race, and no origin. Anonymous is a force and as such, simply is. Anonymous is not I, you, or we. Anonymous is all without name, blame, and restraint. Anonymous cannot be hurt, damaged, or stopped.

(page name: The Anonymous; page ID: 16167528389853; post ID: 43026329370639; date: 2 November 2012)

Anonymous has no will, no purpose, no predefined direction to follow. This, instead of representing a weakness, is converted into the very same conditions of its indestructability: you cannot chase that which does not follow a route.

The will of Anonymous is unclassifiable and therefore is unconquerable. This is the root of its purpose. For kingdoms and armies whose objectives and goals are obvious are easily manipulated and destroyed. When you know what your peers want or need, you know exactly how to control them. Anonymous needs nor wants anything. It has no moral brackets that one could use to predict it's behavior. Its transparent unpredictability is an impenetrable shield which no manipulative force can hinder or tame

(page name: Anonymous; page ID: 1700443796880455; post ID: 174007907625026o; date: 30 May 2016)

Anonymous is never the same, as Anonymous is constantly evolving and redefining. Its dynamic character is not just a matter of natural motion of everything, but a productive flow which is endorsed to access a source of constant energy.

Chaos is change and change is life. Anonymous is in constant flux. It is the embodiment of change. When Anonymous demands justice, it asks not for stagnant order, but disarray and destruction. Anonymous seeks to set fire the overgrown fields of the social order so that their ashes may nutrient the growth of healthy plants.

(page name: Anonymiss Germany; page ID: 36643559355774; post ID: 46056295747833; date: 28 February 2016)

The possibility of negating its own propositions and attending to any conflicting goal, according to some Anons, is not an expression of disfunction, but rather the result of a deliberate plan: Anonymous is incoherent by definition.

Once an individual attempts to act as, or act for, the many, Anonymous is no longer the incoherent machine it is intended to be.

(page name: Anonymous; page ID: 14033595149; post ID: 122409335770; date: 19 September 2009)

Anonymous continuously contravenes its own rules and contradicts itself. However, this is nothing that Anons themselves have not embedded in their self-representation. What can be seen, on a certain level, as a fake manifestation of the real anonymous, on another level of abstraction is explicitly recognized as a constitutive element. Anonymous is undefinable by definition. It is, in Anons' words, a legion: not simply one unified entity, nor simply the juxtaposition of many groups. 
In other words, it is a multiplicity: it vividly exists in a fractional dimension, as 'more than one less than many' (Mol, 2002). Anonymous, however, is also a family: the many diverging opinions and the recurrent infights do not compromise a strong sense of 'we'-ness and unity, thus expressing the existence of a collective identity (Kavada, 2015; Melucci, 1996), although undoubtedly sui generis. Anonymous has a peculiar ontological character, since it is not a group in the conventional sense, nor even something that actually exists: it is an abstract idea that can be incarnated in different forms. Conversely, Anonymous exists as a virtuality: it is real but not actual, because it can be actualized in different, contingent forms ((Deleuze, 1988). The possibility to re-actualize a pure virtuality in radically different instances is what makes so evident Anonymous' multiplicity status and its role of incoherent machine: the heterogeneity and contradictions between its manifestations, as well as the systematic mismatch between its declarations and its behavior, are anticipated in its own definition.

Again, when translated in synthetic propositions, the answers to the question 'who is Anonymous?' that we can derive from Anons' self-definitions are paradoxical and/or tautological: 'Anonymous is one, Anonymous is many'. 'Anonymous does not exist, Anonymous simply is'. 'Anonymous is everything, Anonymous is nothing'. 'Anonymous has no will, Anonymous wants everything'. 'Anonymous is always the same because Anonymous is never the same'. 'Anonymous is intended not to be intended'.

\section{Complexity Unveiled: From (Radical) Reflexivity to (Radical) Recursivity?}

When discussing the problems of applying the term identity to dynamic, composite actors such as social movements, Melucci argues that 'when requested its identity, a collective actor (excluding the extreme, completely schizophrenic situation) is able to provide an answer through its many mouths in a definite way' (Melucci, 1996, p. 86). The previous sections have, in turn, assessed how Anonymous adhere to this extreme, completely 'schizophrenic' situation, and confirmed that, being this the case, the many mouths of the collective actor are unable to answer in a definite way to the question: who are you? These answers are generally maintained as self-referential, rather than resolved in non-recursive forms.

The previous sections have reported statements that have already been widely acknowledged by poststructuralist theories reflecting on the complexity of society: any group is in a permanent state of group formation (Latour, 2005); any entity is the actualization of a virtual multiplicity (Deleuze, 1988); any copy bears a counter-intuitive relation towards the notion of original (Baudrillard, 1994); any object exists in a fractional dimension at the intersection of multiple realities (Mol, 2002). However, the investigation of the self-definitions of Anonymous shows a homology between these complex propositions developed by intellectuals to deconstruct the appearances of reality and analogously complex propositions developed by social movements in the act of self-description.

It is impossible to define Anonymous in ostensive terms from what it does, believes or says, because of the lack of even a residual core of coherence. Trying to provide an extensional definition of Anonymous is challenging because of the widespread inauthenticity claims. Providing an intensional definition is alike problematic, since no essential traits exist that could be made explicit. However, following Anonymous' own declarations, many definitions of who is Anonymous are implicitly or explicitly stated. The counterintuitive aspect, though, is that these definitions are often expressed in terms of tautologies ('Anonymous is everyone because everyone is Anonymous') or of paradoxes ('everyone is Anonymous, because Anonymous is no one'). The systematic mismatch 
between what Anonymous says and what Anonymous does is a further element of contradiction; coupled with the deliberate mission of being an incoherent machine, however, this mismatch generates a paradox of the sort: 'Anonymous does not what Anonymous says'; 'Anonymous says that Anonymous does not what Anonymous says'; 'Anonymous does what Anonymous says', etc. Anonymous, in other words, lies in an ontological paradox which allows it to convert the very same conditions of its dismantling into the conditions of its persistence.

Anonymous can only be defined in self-referential terms precisely because non-essentiality has been incorporated in its essentiality, inauthenticity in its authenticity. Anonymous is not only a multiplicity; it defines itself as an incoherent machine. Anonymous does not contradict itself even (especially) when it contradicts itself. Its complex character is not confined to its inner logic of reproduction, instead is brought to the surface, incorporated in its representations; consequently, the recursive loop upon which any social system is based is never cut, as recursion itself is made explicit rather than hidden.

It is useful to introduce a pragmatic distinction between reflexivity and recursivity. Whereas both are self-referential processes, thus signals of systems acting upon themselves, reflexivity is often understood as a relation between series of self-observations, in which each step incorporate a synthesis of previous observations; we understand here recursivity, instead, as a relation between self-observations at different degrees of abstraction, in which each observation is subsumed but not synthesized by each level of abstraction. The need for this distinction comes from the recognition that whereas radical reflexivity is an acknowledged property of late modernity (Beck et al., 1994; Dean, 2009) and, more specifically, of 'new social movements', Anonymous' relation to self-reference is quite distinctive. The feminist movement, for example, was never a monolith but an open field of elaboration, precisely because of its emphasis on reflexivity (Melucci, 1996). The anti-globalization movement transcended internal incoherence through 'reflexive framing', the iterative renegotiation of meaning (Chesters \& Welsh, 2005). In this sense, reflexivity refers to the capacity of subjects to retroductively question their own identity, in the process synthesizing new definitions. In contrast, recursion is recalled here as the process of abstracting a meta-level of identity, one that does away with the necessity of synthetic definitions. Anonymous is who is Anonymous; Anonymous is not who Anonymous says Anonymous is; Anonymous is everyone and no one; Anonymous, after all, does not even exist.

According to Dean, the rise of communicative capitalism marks the intensification of the circulation of symbols which, in turn, reduce their efficiency in anchoring meaning and identities (Dean, 2009). This represents a condition in which 'reflexivity, then, 'goes all the way down' as we are captured in continuous loops of self-questioning and doubt' (Mumby, 2016, p. 891). Anonymous, on the contrary, seems to create permanent opportunities for action and identification by embracing what we can call 'radical recursion' (Rosen, 2004). What 'goes all the way down' in these cases is not reflexivity, but recursivity: the impossibility of action that would follow from endless contingency, doubts and reflections is ultimately surpassed by the abstraction of a higher-order level of observation. Whereas conventional organizations 'are driven by the continuous need to deparadoxify their inherent contingency' (Schoeneborn, 2011, p. 682), Anonymous perpetuate its immanent contingency by embracing its paradoxical foundations. In this way contradiction, a source of schismogenesis, is sublimated in paradox, a source of autopoiesis. Despite the two terms being often conflated in common language, indeed, 'a paradox is not a contradiction. A paradox abolishes contradiction. It does not negate, it compounds.' (Massumi, 1992, p. 21). 
This leads back to Luhmann's observation that modern societies need to deparadoxize their foundations through ideologies. Whereas in segmentary societies the problem of self-description is solved on a rather immediate level, close to everyday experience, with the advent of modernity 'society responds to the loss of its natural and unchallenged representation by stating the problem of identity in a more abstract way' (Luhmann, 1988, p. 27). Anonymous seems to move further in this abstraction process, pushing the 'modernist' reproduction of the identity of a system towards a further degree of abstraction, one that Luhmann's could only postulate and not observe:

fundamentally different forms of societal self-descriptions have not been established [...] In the long run, it may be possible that second-order systems-theoretical observations (that is, observations and descriptions of societal self-observations and self-descriptions) will yield very different results. (Luhmann, 1992, p. 29)

Anonymous resembles this set of second-order observations that moves the logic of the autopoiesis of social systems to a new level. We could then interpret Anonymous following Melucci's conceptualization of social movements as 'prophets' (Melucci, 1996): a fringe social formation announcing yet-to-be-consolidated patterns of reproduction, thus opening endless opportunities for action and identity in a era of generalized doubt and impasse. The price to pay for such a transition, this case seems to suggest, is being able to sustain our gaze into the abyss, embracing the paradox above which we all lie.

\section{References}

Ahuvia, A. (2001). Traditional, Interpretive, and Reception Based Content Analyses:

Improving the Ability of Content Analysis to Address Issues of Pragmatic and

Theoretical Concern. Social Indicators Research, 54(2), 139-172.

https://doi.org/10.1023/A:1011087813505

Andersen, N. Å. (2011). Conceptual history and the diagnostics of the present. Management

\& Organizational History, 6(3), 248-267. https://doi.org/10.1177/1744935911406152

Bateson, G. (1972). Steps to an Ecology of Mind: Collected Essays in Anthropology,

Psychiatry, Evolution, and Epistemology. University of Chicago Press.

Baudrillard, J. (1994). Simulacra and Simulation. University of Michigan Press.

Beck, U., Giddens, A., \& Lash, S. (1994). Reflexive Modernization: Politics, Tradition and

Aesthetics in the Modern Social Order. Stanford University Press.

Bennett, W. L., \& Segerberg, A. (2012). The Logic of Connective Action. Information,

Communication \& Society, 15(5), 739-768.

https://doi.org/10.1080/1369118X.2012.670661

Chesters, G., \& Welsh, I. (2005). Complexity and Social Movement(s): Process and

Emergence in Planetary Action Systems. Theory, Culture \& Society, 22(5), 187-211.

https://doi.org/10.1177/0263276405057047 
Coleman, G. (2013). Anonymous in Context: The Politics and Power behind the Mask. Centre for International Governance Innovation.

https://www.cigionline.org/publications/anonymous-context-politics-and-power-behind-mas $\underline{\mathrm{k}}$

Coleman, G. (2015). Hacker, Hoaxer, Whistleblower, Spy: The Many Faces of Anonymous (Reprint edition). Verso.

Dean, J. (2009). Democracy and Other Neoliberal Fantasies: Communicative Capitalism and Left Politics. Duke University Press.

Deleuze, G. (1988). Bergsonism. Zone Books.

Deleuze, G., \& Guattari, F. (1987). A Thousand Plateaus: Capitalism and Schizophrenia. University of Minnesota Press.

Dobusch, L., \& Schoeneborn, D. (2015). Fluidity, Identity, and Organizationality: The Communicative Constitution of Anonymous. Journal of Management Studies, 52(8), 1005-1035. https://doi.org/10.1111/ioms.12139

Fuchs, C. (2013). The Anonymous movement in the context of liberalism and socialism. 5(2), $345-376$.

Kavada, A. (2015). Creating the collective: Social media, the Occupy Movement and its constitution as a collective actor. Information, Communication \& Society, 18(8), 872886.

Latour, B. (2005). Reassembling the social: An introduction to actor-network-theory / Bruno Latour. Oxford University Press.

http://proxy.uba.uva.nl:2048/login?url=http://search.ebscohost.com/login.aspx?direct=true\& $\underline{\mathrm{db}=\text { nlebk\&AN}=211608 \& \text { site }=\text { ehost-live }}$

Luhmann, N. (1988). Tautology and Paradox in the Self-Descriptions of Modern Society (S. Fuchs, Trans.). 6(1), 21-37.

Luhmann, N. (1992). What is Communication? Communication Theory, 2(3), 251-259. https://doi.org/10.1111/j.1468-2885.1992.tbooo42.X

Luhmann, N. (1995). Social Systems. Stanford University Press.

Massumi, B. (1992). A User's Guide to Capitalism and Schizophrenia: Deviations from Deleuze and Guattari. MIT Press. 
Maturana, H. R., \& Varela, F. J. (1991). Autopoiesis and Cognition: The Realization of the Living. Springer Science \& Business Media.

McDonald, K. (2006). Global movements: Action and culture. Blackwell Pub.

Melucci, A. (1996). Challenging Codes: Collective Action in the Information Age. Cambridge University Press.

Mol, A. (2002). The Body Multiple: Ontology in Medical Practice. Duke University Press.

Monterde, A., Calleja-López, A., Aguilera, M., Barandiaran, X. E., \& Postill, J. (2015). Multitudinous identities: A qualitative and network analysis of the $15 \mathrm{M}$ collective identity. Information, Communication \& Society, 18(8), 930-950.

https://doi.org/10.1080/1369118X.2015.1043315

Mumby, D. K. (2016). Organizing beyond organization: Branding, discourse, and communicative capitalism. Organization, 23(6), 884-907.

https://doi.org/10.1177/1350508416631164

Olson, P. (2013). We Are Anonymous. Random House.

Palys, T. (2008). Purposive Sampling. In The SAGE Encyclopedia of Qualitative Research Methods (pp. 698-698). SAGE Publications, Inc. https://doi.org/10.4135/9781412963909

Rieder, B., Abdulla, R., Poell, T., Woltering, R., \& Zack, L. (2015). Data critique and analytical opportunities for very large Facebook Pages: Lessons learned from exploring "We are all Khaled Said.” Big Data \& Society, 2(2), 2053951715614980.

https://doi.org/10.1177/2053951715614980

Rosen, S. M. (2004). What is Radical Recursion? SEED Journal, 4(1), 38-57.

Schoeneborn, D. (2011). Organization as Communication: A Luhmannian Perspective.

Management Communication Quarterly, 25(4), 663-689.

https://doi.org/10.1177/0893318911405622

Uitermark, J. (2017). Complex contention: Analyzing power dynamics within Anonymous.

Social Movement Studies, 16(4), 403-417.

https://doi.org/10.1080/14742837.2016.1184136 
Wiedemann, C. (2014). Between swarm, network, and multitude: Anonymous and the infrastructures of the common. Distinktion: Journal of Social Theory, 15(3), 309-326. https://doi.org/10.1080/1600910X.2014.895768 